\section{Intracortical osteosarcoma: Is MRI useful?}

Intracortical osteosarcoma is the rarest variant of osteosarcoma, originating from the bone cortex. We describe the imaging features, with special emphasis on MRI, of an intracortical osteosarcoma occurring in the tibia of a young female. The value of fat-saturated sequences in the local staging of this tumor, especially their sensitivity to demonstrate subtile peritumoral medullary and soft tissue oedema - not appreciated with conventional imaging techniques - is discussed.

\section{Case report}

A 34-year-old female was admitted at our department with local tenderness at the right lower leg since 6 months.

Radiographs of the right tibia revealed an ovoid intracortical lucency in the ventromedial tibial diaphysis, surrounded by thickened and sclerotic cortical bone (Fig. 1).

On CT, the central intracortical lucency was of soft tissue attenuation, with some internal high density septa and focal cortical penetration at the periosteal side of the lesion (Fig. 2), while the surrounding cortex was thickened.

On MRI, the center of the lesion was of intermediate signal intensity on $\mathrm{T}_{1}$ weighted images (Fig. 3 a) and hyperintense on fat-saturated $\mathrm{T}_{2}$-weighted images (Fig. $4 \mathbf{a}-\mathbf{b}$ ). There was enhancement of the central component, especially at the periphery, after intravenous gadolinium administration (Fig. 3 b). The peripheral cortical sclerosis was hypointense on all pulse sequences. Peritumoral medullary and juxtacortical oedema was demonstrated on fat-saturated $\mathrm{T}_{2}$-weighted images (Fig. $4 \mathbf{a}-\mathbf{b}$ ).

The radiological differential diagnosis included adamantinoma, ossifying fibroma, a diaphyseal variant of osteoblastoma and intracortical osteosarcoma. An intracortical abscess was less probable, because of the absence of inflammatory parameters.

A surgical biopsy of the osteolytic center of the lesion was performed, and histological examination of the biopsy specimen showed features of an osteosarcoma, containing a matrix with os- teoid material and sheets of polygonal cells possessing prominent nucleoli and moderate amounts of eosinophilic cytoplasm. Sparsely mitotic figures were seen.

The patient was treated with three sessions of preoperative chemotherapy, consisting of a combination of doxorubicin and cisplatinum, every two weeks, followed by en-bloc resection of the tumor. Histopathological examination of the resected specimen failed to demonstrate any residual tumor activity in the central cortical lucency, probably due to a good response to chemotherapy. There was a marked residual reactive ossification within the adjacent cortex.

\section{Discussion}

Intracortical osteosarcoma (ICOS) is the rarest variant of osteosarcoma (Yang $\mathrm{SH}$ et al., J Formos Med Assoc 2000; 99: 721), with only seventeen previous cases reported to date. It is typically confined to the cortex of the diaphysis, in contradistinction to conventional osteosarcoma which has a typical medullary location within the metaphyses of long bones. Among the eighteen reported cases, nine lesions were located in the femur, while eight cases (including our case) were confined to the tibial cortex. Only one of the reported cases was located in the humerus (De Boeck $\mathrm{H}$ et al., Acta Orthop Belg 1998; 64:96). The patient age ranged from 9 to 43 years, with most lesions occurring in the second to third decade.

Clinical manifestations include pain, local tenderness or a palbable mass over the affected bone for less than 1 year's duration.

Standard radiographs typically show an intracortical lucency, ranging from 1 to $4 \mathrm{~cm}$ in diameter, within the diaphysis, surrounded by thickened sclerotic cortical bone (Griffith JF et al., Skeletal Radiol 1998; 27: 228). This may falsely suggest benign entities, like fibrous cortical defect, intracortical abscess, fibrous dysplasia, ossifying fibroma or a diaphyseallocated osteoblastoma (Rosenberg ZS et al., AJR 1995; 165: 1209). Another differential diagnosis is adamantinoma, which

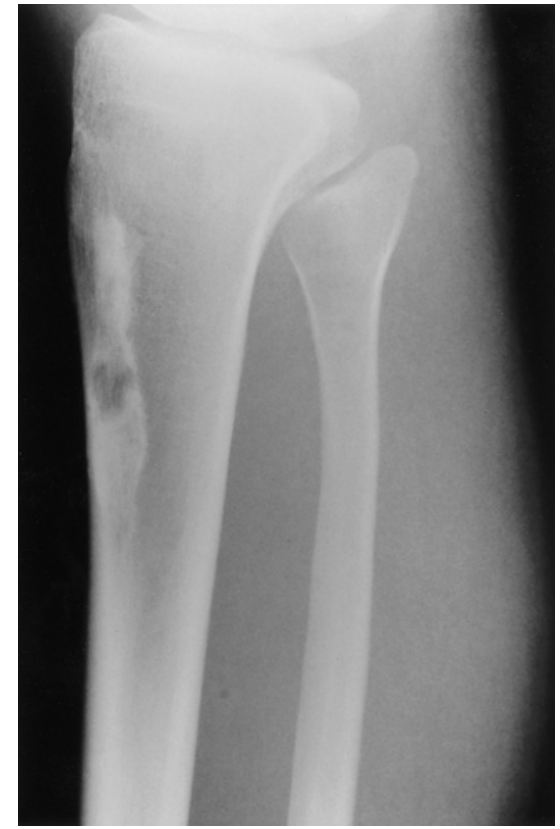

Fig. 1 Lateral radiograph of the right tibia. A broad-based area of cortical sclerosis and thickening, encompassing a central lytic area, is seen at the anterior aspect of the proximal tibial diaphysis.

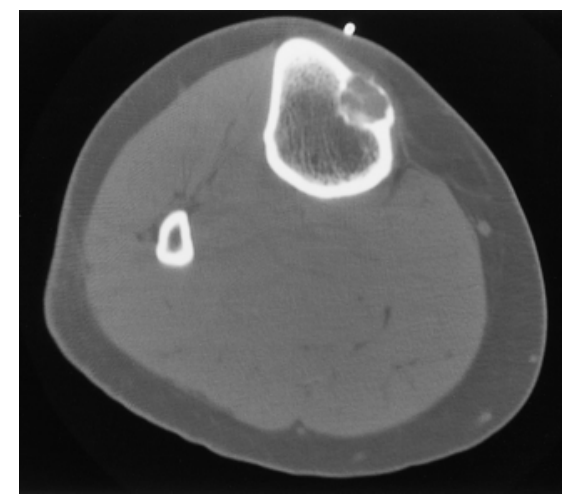

Fig. 2 CT scan at the level of the central lucency. Focal cortical penetration at the periosteal side of the central intracortical lucency. An internal high density septum is seen as well.

has also a predilection for the tibial diaphyseal cortex.

CT is useful in demonstrating cortical penetration of the central intracortical lucency, which contains rarely matrix calcifications, and to define the extent of the surrounding cortical sclerosis.

MR imaging has only been described in 4 previous cases of ICOS. On $\mathrm{T}_{1}$-weighted images, the central cortical component was isointense to muscle and heterogeneously hyperintense on $\mathrm{T}_{2}$-weighted 

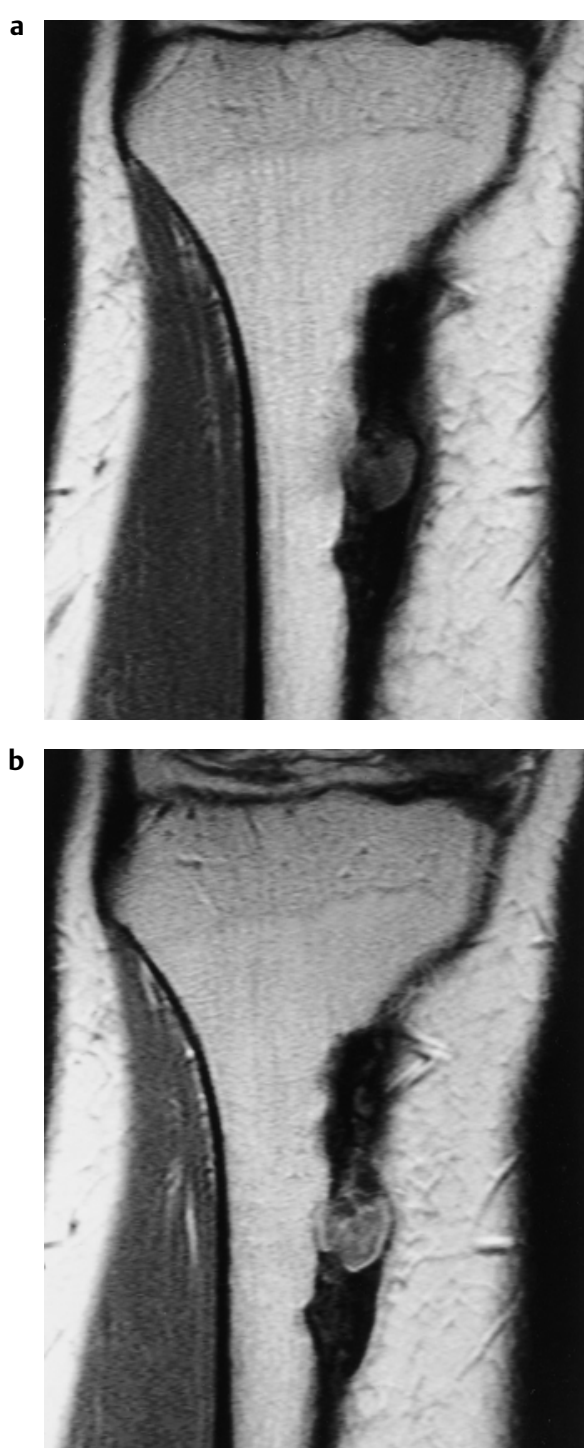

Fig. 3 Coronal $\mathrm{T}_{1}$-weighted $\mathrm{MR}$ image of the tibia before and after intravenous gadolinium contrast administration. The central intracortical tumor is isointense to muscle (a), and is surrounded by a thickened cortex. There is peripheral enhancement of the central tumor component (b). The adjacent medullary bone is slightly hypointense (a).

images. Only in our case, gadoliniumchelates were intravenously administrated, which resulted in strong peripheral enhancement of the central tumor nodule. The surrounding cortical sclerosis was hypointense on all pulse sequences. In only one previously reported case (Griffith JF et al., Skeletal Radiol 1998; 27: 228), as well as in our case, was peritumoral medullary and iuxtacortical oedema seen. This finding was particularly well demonstrated on fat-saturated $\mathrm{T}_{2}$-weighted images, and far less obvious on spin-echo $\mathrm{T}_{1}$ -

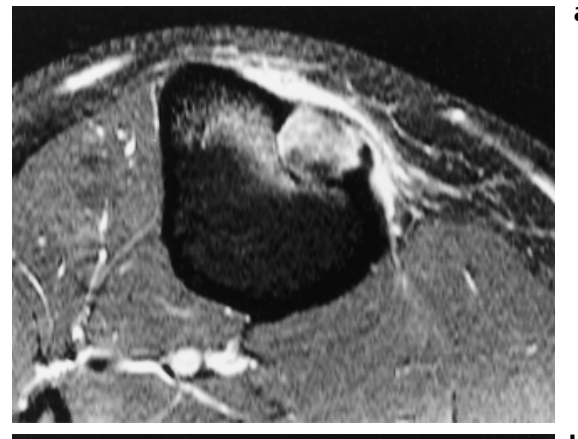

modality of choice for local tumor staging.

Because the tumor can mimic several other benign tumors, biopsy of the lesion however is mandatory, to confirm the diagnosis histologically.

If an ICOS is suspected on conventional radiography, we recommend MRI as the next imaging modality for further diagnostic work-up and local staging, repla-

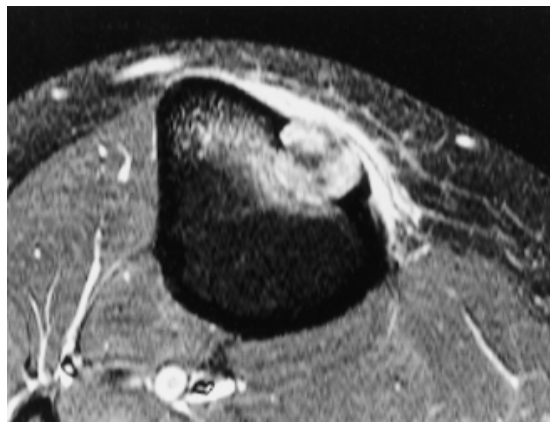

Fig. 4 Axial fat-saturated $T_{2}$-weighted $M R$ image at two different levels of the central tumor component. Prominent medullary and soft tissue oedema is seen.

weighted images. This perilesional oedema is in contradiction with the classical description of ICOS, which confines the tumor solely within the cortical bone and grossly involving neither the medullary space nor the soft tissues (Jaffe HL. Bull Hosp Jt Dis 1960; 21: 189). The peritumoral oedema probably reflects the increased sensitivity of fat-saturated sequences for oedema, rather than being a new feature of the tumor. However, this illustrates very well that because of the high sensitivity of fat-saturated sequences for medullary and juxtacortical involvement, MRI should be the imaging cing CT, unless a CT-guided biopsy and not a surgical biopsy is planned.

Treatment consists of en-bloc resection, with adjuvant chemotherapy (Yang SH et al., J Formos Med Assoc 2000; 99: 721).

In conclusion: ICOS is the rarest variant of osteosarcoma, with a typical location in the diaphyseal cortex of the femur or tibia. Radiologically, it may mimic a benign lesion. Although it was originally believed to represent a pure cortical lesion, neither involving the medullary bone nor the soft tissue, fat-saturated sequences can clearly demonstrate peritumoral, medullary and soft tissue oedema, not appreciated on conventional imaging techniques.

\section{Take home points: Intracortical Osteosarcoma}

1. Typical location within the diaphyseal cortex of femur or tibia

2. Radiographs may mimic benign conditions

3. MRI with fat saturation is very useful for demonstration of perilesional oedema, and local tumor staging, respectively

F. Vanhoenacker, L. H. De Beuckeleer, A. M. De Schepper, Edegem, Belgien 